\title{
The control of stimulus-driven saccades is subject not to central, but to visual attention limitations
}

\author{
Elena Carbone and Werner X. Schneider \\ Bielefeld University, Bielefeld, Germany
}

\begin{abstract}
In three experiments, we investigated whether the control of reflexive saccades is subject to central attention limitations. In a dual-task procedure, Task 1 required either unspeeded reporting or ignoring of briefly presented masked stimuli, whereas Task 2 required a speeded saccade toward a visual target. The stimulus onset asynchrony (SOA) between the two tasks was varied. In Experiments 1 and 2, the Task 1 stimulus was one or three letters, and we asked how saccade target selection is influenced by the number of items. We found (1) longer saccade latencies at short than at long SOAs in the report condition, (2) a substantially larger latency increase for three letters than for one letter, and (3) a latency difference between SOAs in the ignore condition. Broadly, these results match the central interference theory. However, in Experiment 3, an auditory stimulus was used as the Task 1 stimulus, to test whether the interference effects in Experiments 1 and 2 were due to visual instead of central interference. Although there was a small saccade latency increase from short to long SOAs, this difference did not increase from the ignore to the report condition. To explain visual interference effects between letter encoding and stimulus-driven saccade control, we propose an extended theory of visual attention.
\end{abstract}

Because visual acuity is high only within the small foveal area, humans need to sample their visual environments via saccadic eye movements. But how do we determine the target for future saccades? A number of behavioral (e.g., Deubel \& Schneider, 1996) and single-cell studies (e.g., Kustov \& Robinson, 1996; a recent review can be found in Awh, Armstrong, \& Moore, 2006) support the notion that visuospatial attention plays an important role for "where to look next." Strictly speaking, it is assumed that an attention shift precedes the execution of a saccade (e.g., Findlay, 2009; Schneider, 1995). Deubel and Schneider, for example, showed that letter discrimination improves at the saccade target location just before the execution of the saccade. Similar results have been found for reflexive saccades (Schneider \& Deubel, 1995, 2002).

Much less is known about the relationship between eye movement control and a second major class of attentional processes that refer to central transmodal processing (e.g., Jolicœur, 1998). Such central attention processes are typically studied in dual-task paradigms such as the attentional blink (AB) or the psychological refractory period (PRP) paradigms. In the $\mathrm{AB}$ paradigm, a second target in a rapid succession of visual displays is missed when it follows the first with a short delay (e.g., Chun \& Potter, 1995; Jolicœur, 1998). In the PRP paradigm, response time (RT) to a second stimulus is prolonged when it follows a first with a short delay (e.g., Pashler, 1994). Results of AB and PRP studies have been explained by the central interfer- ence theory (CIT; e.g., Jolicœur, 1998, 1999a; Jolicœur \& Dell'Acqua, 1998). According to CIT, cognitive processes such as response selection within the PRP or short-term consolidation (STC) within the AB paradigm require central processing. These central attention processes cannot be executed simultaneously. Thus, when one is occupied with one central process such as STC for one stimulus, central processing of a trailing stimulus has to wait; that is, response selection or consolidation for this stimulus is delayed (Jolicœur, 1998, 1999a).

On the surface, a close relationship between central attention processes and the control of saccadic eye movements seems rather unlikely, particularly when one refers to reflexive eye movements that are triggered by the onset of a stimulus and targeted toward that stimulus. In line with these considerations, Pashler, Carrier, and Hoffman (1993) did not find the usual PRP effect for eye movements in a dual task when the participants made a saccade toward single transients. Instead, the RT prolongation was very modest.

However, with the close link between eye movements and visual attention in mind, indirect hints already make an involvement of central attention processes in saccadic control probable. These studies have shown that visual attention for one stimulus interferes with central processing of another stimulus. For example, in a variant of the $\mathrm{AB}$ paradigm, Dell'Acqua, Sessa, Jolicœur, and Robitaille (2006) investigated two components of event-related po-

E. Carbone, elena.carbone@uni-bielefeld.de 
tentials (ERPs): the N2pc, an ERP component that reflects covert visuospatial attention; and the SPCN, a component that reflects neural activity related to the maintenance of information in visual short-term memory. A first and a second target (T1 and T2) were embedded in two alphanumeric streams shifted to the left and to the right relative to a fixation cross. T1 had either to be reported or to be ignored, whereas T2 always had to be reported. When T1 was irrelevant, the authors found $\mathrm{T} 2$ to produce a strong $\mathrm{N} 2 \mathrm{pc}$, as well as a subsequent SPCN. When T1 and T2 had to be reported, both components for T2 were suppressed. The authors concluded that those mechanisms responsible for visual-spatial attention are disturbed by contemporary encoding of information into short-term memory (STM). In line with this conclusion, Brisson and Jolicœur (2007) found the N2pc for a stimulus to be reduced when observers were occupied with the central processing of another stimulus, using a cross-modal PRP paradigm.

Thus, more direct evidence for shared attentional processes between saccade target (ST) selection and central processes is needed. Adapting a dual-task procedure used by Jolicœur and Dell'Acqua (1998), a first visual stimulus (T1) has to be encoded and later reported without time pressure. With varying stimulus onset asynchronies (SOAs) relative to T1 (short SOA of $200 \mathrm{msec}$ or long SOA of $800 \mathrm{msec}$ ), an ST is presented, and the participants have to execute a saccade toward that stimulus instantly. If ST selection draws on the same resources as the central attention process STC, prolonged saccade latencies are expected with short SOAs, since STC would not have been finished, as opposed to long SOAs.

In addition, we tried to manipulate the duration of STC. Jolicœur and Dell'Acqua (1998) have shown in a series of experiments that an increase of the number of memory items in Task 1 led to an increase of RT in Task 2 and proposed that the duration for STC increased with an increasing number of items and that response selection for Task 2 was delayed more strongly in cases with a large number of items. More precisely, T1 consisted of either one or three briefly presented characters for later unspeeded STM-based report. The second stimulus (T2) was a tone that required a speeded choice reaction. The SOA between T1 and T2 (350-1,600 msec) was varied. As predicted by CIT, the authors found increasing RTs for T2 with decreasing SOAs. Importantly, this increase in manual response latency for the three-character condition was stronger than that for the single-character condition.

Summing up, in order to test whether central attention is linked to the control of reflexive saccades, we utilized Jolicœur and Dell'Acqua's (1998) dual-task paradigm and the manipulation of the number of items to be remembered. Decisively, we replaced manual responses by stimulusdriven saccades. If the link indeed exists, we would expect the same particular pattern of interference effects to show up in saccadic latencies as those found for manual responses.

\section{EXPERIMENT 1}

We predicted an increase of saccade latency for T2 at short SOAs, as compared with long SOAs. Importantly, this latency increase should be more pronounced when the number of items is increased from one to three items. We also added a control condition with the letters being irrelevant (ignore condition). Since STC is not required here, we expected no difference between SOAs in saccade latency and, in particular, no increase of the latency difference between SOAs with an increasing number of items.

\section{Method}

Participants. Sixteen participants (13 female and 3 male) took part in Experiment 1. Their average age was 26 years. In all three experiments, the participants were students or staff from Bielefeld University and had normal or corrected-to-normal visual acuity. They were not informed about the aims of the experiment.

Apparatus and Stimuli. The experiment was controlled by the Experiment Builder software (SR Research, Canada). The stimuli were displayed on a 19 -in. color monitor with a refresh rate of $100 \mathrm{~Hz}$ and a resolution of $1,024 \times 768$ pixels. The participants' gaze positions were recorded by an EyeLink 1000 tower system (SR Research). The eyetracker's sampling rate was $1000 \mathrm{~Hz}$, and the typical average accuracy lay between $0.25^{\circ}$ and $0.5^{\circ}$. Viewing distance was fixed at $71 \mathrm{~cm}$.

The stimuli were displayed on a black background. The central fixation cross $\left(0.72^{\circ} \times 0.72^{\circ}\right)$ was white, and the target stimuli (T1s) were green consonants $\left(0.42^{\circ} \times 0.48^{\circ}\right)$. In cases with a one-letter stimulus, the consonant was shown at the screen's center. For the three-letters stimulus, two letters were added, shifted by $0.72^{\circ}$ to the left and to the right. Each letter was masked by a white pattern mask $\left(0.66^{\circ} \times 0.66^{\circ}\right)$. The ST was a white cross $\left(0.72^{\circ} \times 0.72^{\circ}\right)$, shifted $4.6^{\circ}$ to the left or to the right with respect to the screen's center. The participants identified the letters via the keyboard.

Design. The within-subjects variables were block type (ignore vs. report of T1), number of letters (one vs. three), and SOA (200 vs. $800 \mathrm{msec}$ ). Block type was blocked (with the block order counterbalanced across the participants), whereas number of letters and SOA were intermixed within the blocks.

Each of the 2 block type $\times 2$ number of letters $\times 2$ SOA trials was shown 24 times, summing up to 192 trials, split up into eight alternating blocks of 24 trials. The experiment started with 10 practice trials for the report condition.

The dependent variables were error rate for the unspeeded letter identification task and saccade latency for the speeded saccade task. We defined an error in Task 1 as a trial on which at least one letter was reported incorrectly. The saccade latency was the time interval between the onset of the ST and the start of the first saccade that left the fixation area (circle with a radius of $0.86^{\circ}$ around the fixation cross's center).

Trials meeting one of the following three criteria were excluded from the saccade latency analysis: (1) Trials with errors in the letter identification task (Task 1 error criterion), (2) trials with saccades landing outside the target area of $2^{\circ}$ around the ST's center (spatial inaccuracy criterion), and (3) trials with saccade latencies exceeding a range of two standard deviations around the individual participant's mean latency or saccades that were definitely anticipatory in nature ( $<80 \mathrm{msec}$; latency outlier criterion).

Procedure. The experiment began with a written instruction, and each block was preceded by a 9-point calibration procedure.

Each trial started with the presentation of the fixation cross. After a fixation duration of $400 \mathrm{msec}$, it vanished, and simultaneously, one or three letters were shown for $120 \mathrm{msec}$. The stimulus was followed by a mask that remained on the screen until the end of the trialthat is, $400 \mathrm{msec}$ after the saccade had been initiated, but at least for $1,400 \mathrm{msec}$, even if the saccade had been initiated earlier. After an SOA of 200 or $800 \mathrm{msec}$ relative to T1, the ST appeared either on the left or on the right. An exemplary trial sequence can be seen in Figure 1.

The participants were supposed to initiate a saccade toward the target as soon as it appeared. In report blocks, they then had to type in the letters and received a feedback about the correctness of their report (" $X$ of $Y$ letters correct"). 


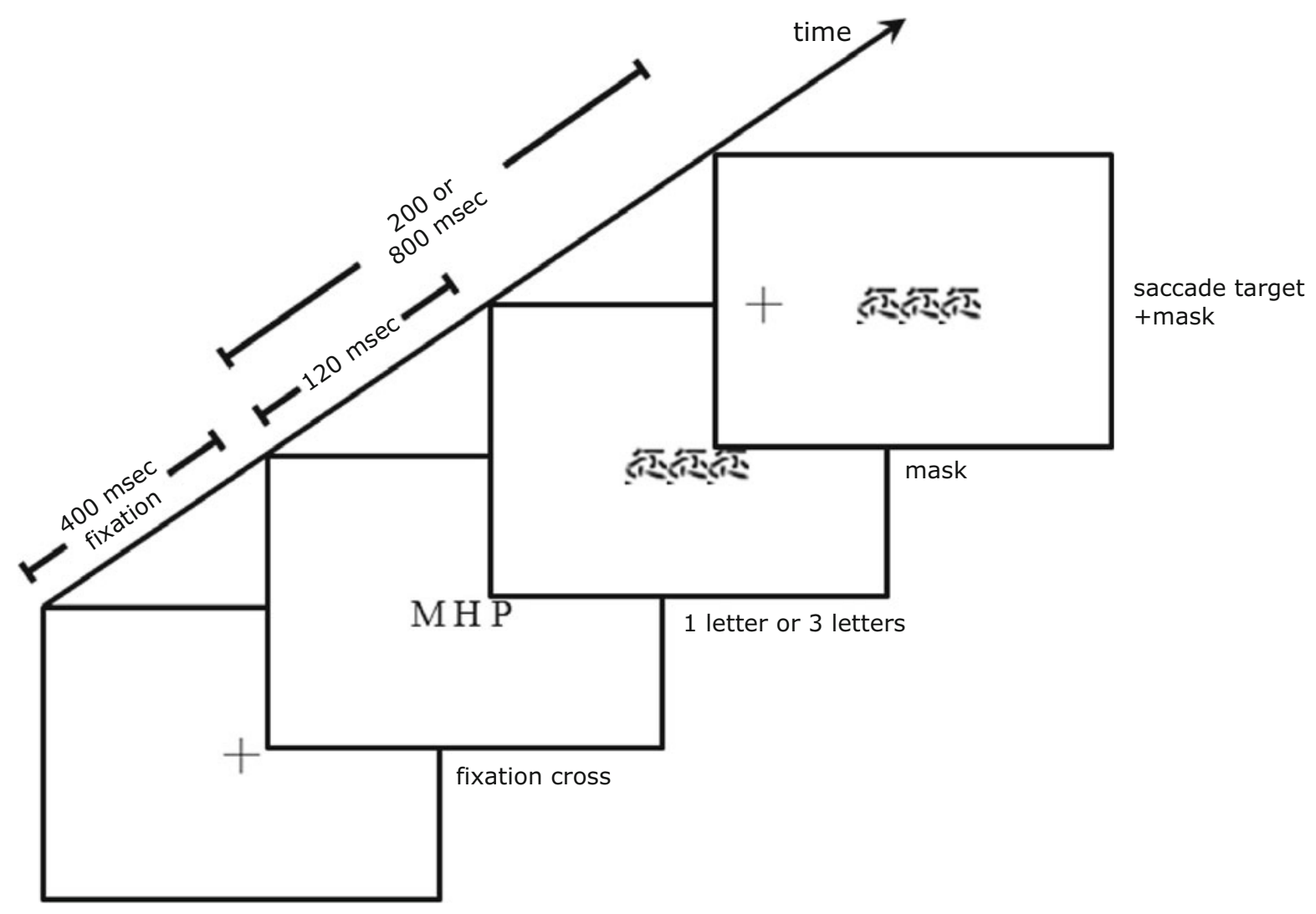

Figure 1. Exemplary stimulus sequence used in Experiments 1 and 2.

\section{Results and Discussion}

Letter identification. Mean letter identification performance was $85.79 \%$. We fed the data into a $2 \times 2$ ANOVA with number of letters (one vs. three) and SOA (200 vs. $800 \mathrm{msec}$ ) as within-subjects variables. The results are depicted in Figure 2.

The analysis revealed a highly significant effect of number of letters, with a higher percentage correct in the one-letter condition $(98.91 \%)$ than in the three-letter condition $(72.66 \%)[F(1,15)=35.514, p<.001]$. The main

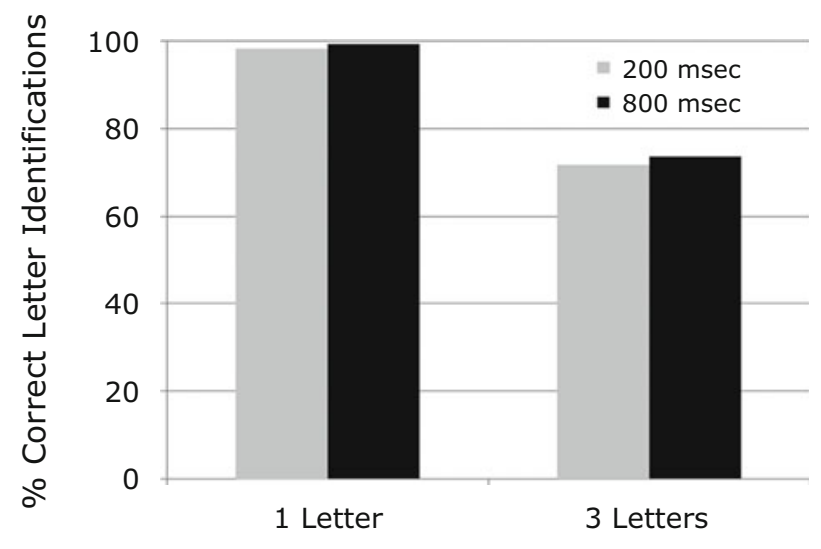

Figure 2. Mean performance in the letter identification task for number of letters (one vs. three) and stimulus onset asynchrony (200 vs. 800 msec) in Experiment 1. effect of SOA and the interaction did not reach significance (both $F \mathrm{~s}<1$ ).

Saccade latency. We excluded all trials that met at least one of the exclusion criteria $(19.21 \%$ of the data).

The remaining data were fed into a $2 \times 2 \times 2$ ANOVA with block type (ignore vs. report), number of letters (one vs. three), and SOA (200 vs. $800 \mathrm{msec}$ ) as within-subjects variables. The results are depicted in Figure 3.

All main effects and interactions reached significance. Saccade latency was longer in report blocks $(284 \mathrm{msec})$ than in ignore blocks $(217 \mathrm{msec})[F(1,15)=18.087, p<$ $.01]$, longer for the three-letter condition $(276 \mathrm{msec})$ than for the one-letter condition $(226 \mathrm{msec})[F(1,15)=9.043$, $p<.01]$, and longer for short SOAs $(294 \mathrm{msec})$ than for long ones $(208 \mathrm{msec})[F(1,15)=45.612, p<.01]$. The two-way interaction between block type and number of letters was highly significant $[F(1,15)=22.760$, $p<.001]$, as were the ones for block type and SOA $[F(1,15)=13.994, p<.01]$ and for number of letters and SOA $[F(1,15)=11.455, p<.01]$. Importantly, the threeway interaction did reach significance as well $[F(1,15)=$ $9.267, p<.01]$.

To investigate whether the pattern of the three-way interaction matched the one found by Jolicœur and Dell'Acqua (1998) for manual responses, we computed differences between the long and short SOAs (Diff ${ }_{\mathrm{SOA}}$ ) for the four block type $\times$ letter number combinations and ran post hoc $t$ tests. We found that for the report condition, Diff $\mathrm{SOA}_{\mathrm{SOA}}$ increased from the one- to the three-letter condition [81.09 


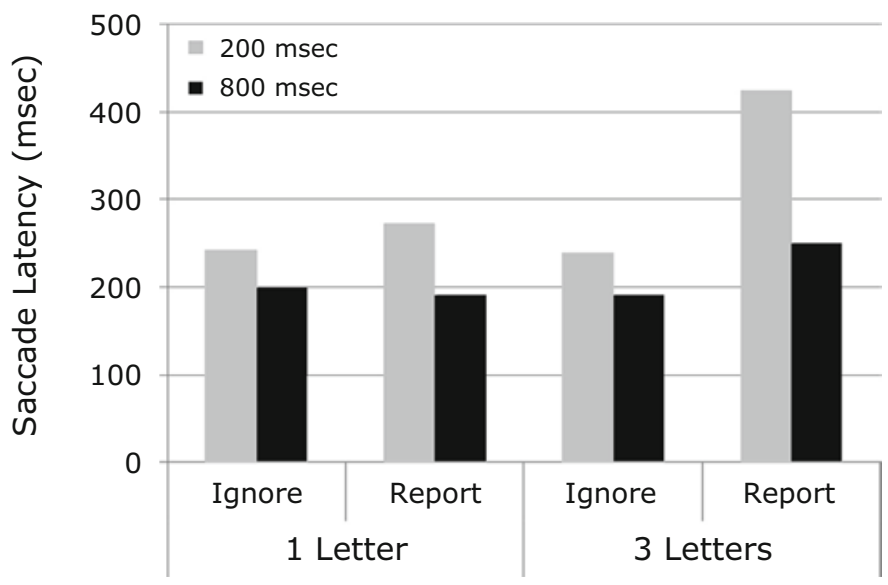

Figure 3. Mean saccade latencies for number of letters (one vs. three), block type (ignore vs. report), and stimulus onset asynchrony (200 vs. 800 msec) in Experiment 1.

for one letter and 174.3 for three letters; $t(15)=3.444$, $p<.01]$, but not for the ignore condition $(43.79 \mathrm{msec}$ for one letter and 47.96 msec for three letters; $t<1$ ). In ad-

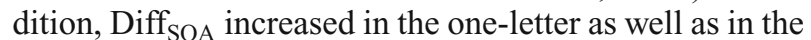
three-letter condition from the ignore to the report condition: from 43.79 to $81.09 \mathrm{msec}$ in the one-letter condition $[t(15)=2.802, p<.05]$ and from 47.96 to $174.3 \mathrm{msec}$ in the three-letter condition $[t(15)=3.636, p<.01]$. However, not predicted by CIT, Diff SOA $_{\text {was significantly }}$ larger than 0 not only in the report condition $[128 \mathrm{msec}$; $t(15)=5.650, p<.001]$, but also in the ignore condition [46 msec; $t(15)=5.990, p<.001]$.

Summing up, the data in Experiment 1 showed that an increase of the number of items led to an increase of the saccade latency difference between SOAs $\left(\right.$ Diff $\left._{\text {SOA }}\right)$. This increase was found only when the items had to be memorized - that is, in the report condition - supporting the idea of shared mechanisms between central attentional process STC and ST selection. In addition to the results matching CIT, the significant latency difference between SOAs in the ignore condition is not predicted by the theory.

Jolicœur and Dell'Acqua (1998) argued that the number of items to be encoded in STM determines the duration of STC. However, with the same presentation duration in the one- and the three-letter condition, as was the case in Experiment 1, task difficulty might be higher for three letters than for one letter (e.g., Kahneman, 1973, stressed the importance of task difficulty for dual-task performance). The large performance difference between the one- and three-letter conditions in Experiment 1 (98.91\% vs. $72.66 \%)$ indicates that this interpretation is indeed plausible. Instead of the number of items, the higher task difficulty might determine the duration of STC. Therefore, it is unclear whether the larger Diff $_{S O A}$ in the three-letter condition was caused by the larger number of letters or by the higher task difficulty. These two alternatives were tested in Experiment 2. To that aim, we equated task difficulty between the one- and the three-letter conditions by adjusting the presentation duration for each participant in both conditions to reach the same memory performance levels in the one- and the three-letter conditions.

\section{EXPERIMENT 2}

For equating task difficulty, we used an adaptive procedure that adjusted the letter presentation durations in the two conditions. If the number of items determines the lon-

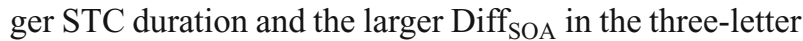
condition, the same result pattern should be found in Experiment 2 as in Experiment 1, since STC should still take longer for three items than for one item. If, however, task difficulty is essential, Diff $\mathrm{SOA}_{\mathrm{S}}$ should be the same in the two letter conditions. In addition, we tried to replicate the unexpected significant Diff $_{\mathrm{SOA}}$ in the ignore condition.

\section{Method}

Participants. Eight participants (5 female and 3 male) with an average age of 31 years took part in Experiment 2.

Apparatus, Stimuli, and Design. The apparatus, stimuli, and design were identical to those in Experiment 1.

Procedure. The procedure was identical to that in Experiment 1, with the following exception. An adaptive method for the letter identification task was used at the beginning of the experiment in order to achieve comparable performance levels in the two letter conditions. We altered the letter presentation duration so as to reach a performance range of $70 \%-85 \%$ correct. Starting from a duration of $60 \mathrm{msec}$ (one letter) and $120 \mathrm{msec}$ (three letters), we ran four blocks of 40 trials each ( 20 trials for each letter condition that were randomly intertwined). After each block, the presentation duration either remained the same or was increased or decreased by 10 or $20 \mathrm{msec}$ according to the deviation from the target performance range.

\section{Results and Discussion}

Adaptive letter presentation duration. The letter presentation durations generated by the adaptive procedure were within a range of $40-80 \mathrm{msec}$ for the one-letter condition and within $90-150$ msec for the three-letter condition. The means were 51 and $114 \mathrm{msec}$, respectively.

Letter identification. The mean letter identification performance was $77.05 \%$. The $2 \times 2$ ANOVA with num- 


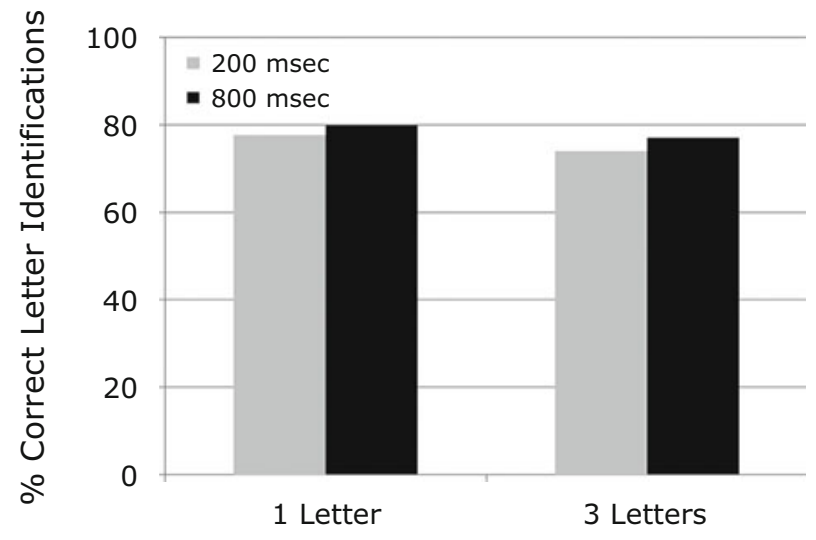

Figure 4. Mean performance in the letter identification task for number of letters (one vs. three) and stimulus onset asynchrony (200 vs. 800 msec) in Experiment 2.

ber of letters (one vs. three) and SOA (200 vs. $800 \mathrm{msec}$ ) as within-subjects variables did not reveal any significant main effect or interaction (all $F_{\mathrm{s}}<1.1$ ). The now nonsignificant effect of the number of letters validated the effectiveness of the adaptive procedure. The results are depicted in Figure 4.

Saccade latency. Of the trials, $24.28 \%$ were excluded according to the criteria defined for Experiment 1.

We ran a $2 \times 2 \times 2$ ANOVA with block type (ignore vs. report), number of letters (one vs. three) and SOA (200 vs. $800 \mathrm{msec}$ ) as within-subjects variables. The results are depicted in Figure 5.

As in Experiment 1, all main effects and interactions reached significance. Saccade latency was longer in report blocks $(313 \mathrm{msec})$ than in ignore blocks $(208 \mathrm{msec})$ $[F(1,7)=24.566, p<.01]$, longer for the three-letter condition $(285 \mathrm{msec})$ than for the one-letter condition $(237 \mathrm{msec})[F(1,7)=8.915, p<.05]$, and longer for short SOAs $(319 \mathrm{msec})$ than for long ones $(203 \mathrm{msec})[F(1,7)=$ $65.146, p<.001]$. The two-way interaction between block type and letter number was highly significant $[F(1,7)=$
$29.915, p<.01]$, as were the ones for block type and SOA $[F(1,7)=47.343, p<.001]$ and for numbers of letters and SOA $[F(1,7)=26.573, p<.01]$. Importantly, the threeway interaction reached significance as well $[F(1,7)=$ $13.799, p<.01]$.

As in Experiment 1, we ran post hoc $t$ tests for Diff SOA $_{\text {A }}$ to clarify the result pattern accounting for the three-way interaction. In the report condition, Diff SOA $_{\text {increased from }}$ the one- to the three-letter condition $[129.29 \mathrm{msec}$ for one letter and $249.71 \mathrm{msec}$ for three letters; $t(7)=4.758, p<$ $.01]$, but not in the ignore condition $(38.69 \mathrm{msec}$ for one letter and $45.81 \mathrm{msec}$ for three letters; $t<1$ ). In addi-

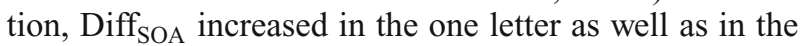
three-letter condition from the ignore to the report condition: from 38.69 to $129.29 \mathrm{msec}$ in the one-letter condition $[t(7)=3.744, p<.01]$ and from 45.81 to $249.71 \mathrm{msec}$ in the three-letter condition $[t(7)=7.229, p<.001]$. The unexpected result of Experiment 1 was also replicated in Experiment 2. Again, Diff SOA $_{\text {as significantly }}$ larger than 0 not only in the report condition $[190 \mathrm{msec}$; $t(7)=8.354, p<.001]$, but also in the ignore condition [42 msec; $t(7)=3.754, p<.01]$.

Summing up, we found an increase of Diff SOA $_{\text {with an }}$ increasing number of items in the report condition only. Again, we also found a significant Diff SOA $_{\text {in }}$ in the ignore condition. Thus, we replicated the results found in Experiment 1 , despite the comparable memory performance levels. These results support the original considerations of Jolicœur and Dell'Acqua (1998) - namely, that the number of items determines the duration of STC and, thereby, the larger Diff SOA $_{1}$ in the three- than in the oneletter condition.

However, a severe objection that could be raised against the interpretation of our data in terms of CIT concerns the stimuli used as T1 and T2. In contrast to Jolicœur and Dell'Acqua (1998), both stimuli were visual, and therefore our interference effects do not need to be transmodal in nature (see Jolicœur, 1999b). Instead, interference could be modality specific. We conducted a third experiment to test the validity of this alternative interpretation.

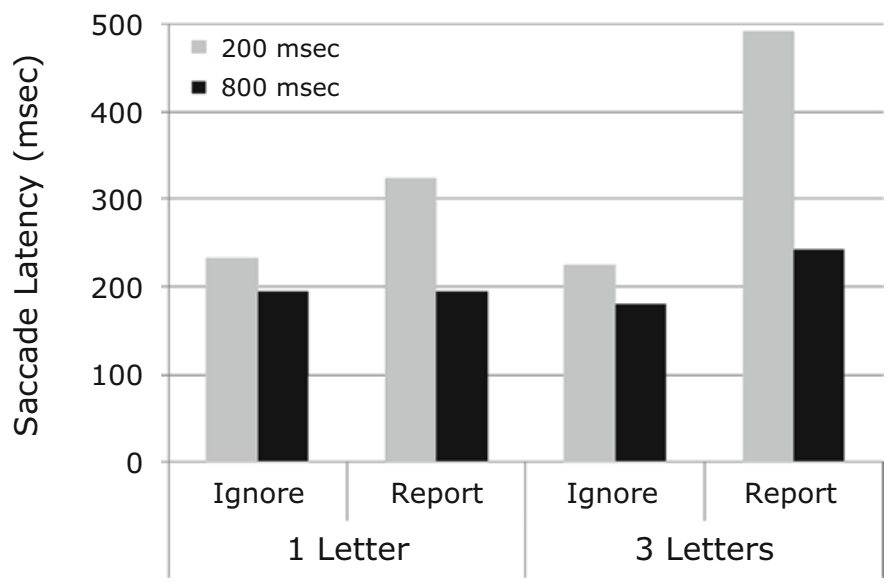

Figure 5. Mean saccade latencies for number of letters (one vs. three), block type (ignore vs. report), and stimulus onset asynchrony (200 vs. 800 msec) in Experiment 2. 


\section{EXPERIMENT 3}

In Experiment 3, we used briefly presented and masked tones, instead of letters, as T1. Since the serial presentation of several tones would require temporal delays between the tones (we did not want to allow for chords), only the one-item condition (i.e., one tone) was used.

If the result pattern found in Experiments 1 and 2 was due to central interference, we would again expect longer saccade latencies for the short- than for the long-SOA condition. This difference should be more pronounced in the report than in the ignore condition. Thus, we predicted a two-way interaction. If, however, the interference was caused by modality-specific interference, the saccade latency prolongation effects should vanish in Experiment 3.

\section{Method}

Participants. Ten female and 5 male participants took part in Experiment 3. Their average age was 24 years.

Apparatus and Stimuli. The apparatus and stimuli were the same as those in Experiment 1, with the following exceptions. Instead of the letters, we used auditory stimuli differing in pitch as T1. The auditory stimuli were two pure tones that differed only slightly in frequency ( 300 vs. $306 \mathrm{~Hz}$ ). They were presented via stereo loudspeakers located left and right of the screen. Each sound was masked by white noise. The participants indicated the pitch of 1 by pressing the left or the right back-button of a gamepad (Microsoft).

Design. The within-subjects variables were block type (ignore vs. report of T1) and SOA (200 vs. $800 \mathrm{msec})$. As in the previous experiments, block type was blocked (with the block order counterbalanced across the participants), and the SOAs where intermixed within the blocks.

Each of the 2 block type $\times 2$ SOA trials was shown 48 times, summing up to 192 trials, split up into eight alternating blocks of 24 trials. The experiment started with 10 practice trials for the report condition.

The dependent variables were error rate for the unspeeded tone pitch discrimination task and saccade latency for the speeded saccade task.

Procedure. The trial procedure was designed so as to ensure maximal comparability with the former letter experiments. Again, each trial started with the presentation of the fixation cross, and after a fixation duration of $400 \mathrm{msec}$, one of the two tones was presented for $90 \mathrm{msec}$. T1 was followed by the auditory mask that was presented until the end of the trial. After an SOA of 200 or $800 \mathrm{msec}$ relative to the $\mathrm{T} 1$ tone, the saccade target appeared either on the left or on the right of the screen. In report blocks, the participants pressed the gamepad button to indicate their tone judgment after the execution of the saccade, and feedback was presented afterward that informed the participants about their discrimination accuracy ("correct" or "incorrect").

\section{Results and Discussion}

Three participants were excluded due to a performance level close to chance in the pitch discrimination task (less than $60 \%$ correct).

Pitch discrimination. The mean pitch discrimination performance for the remaining participants was $74.46 \%$. A paired $t$ test for the two SOAs did not reach significance (74.75\% for the short SOA and $74.17 \%$ for the long SOA; $t<1)$. The results are depicted in Figure 6.

Saccade latency. Of the remaining trials, $19.7 \%$ were excluded according to the criteria defined for Experiment 1 .

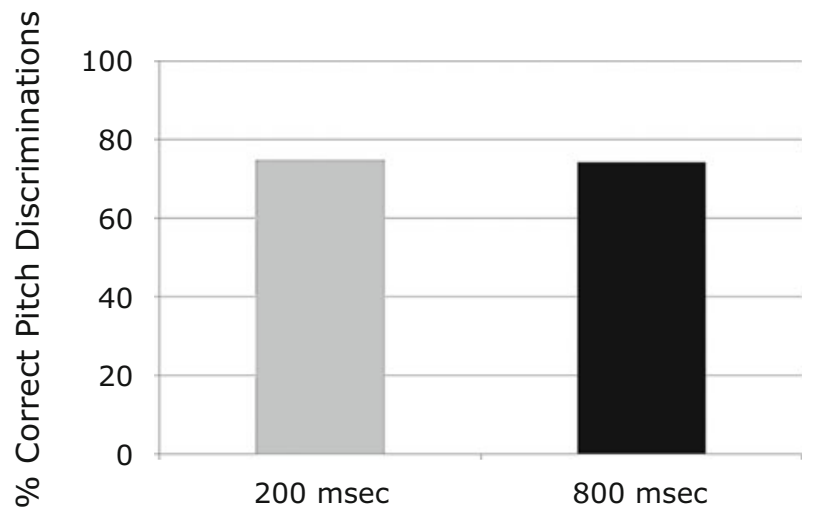

Figure 6. Mean performance in the pitch discrimination task for stimulus onset asynchrony (200 vs. $800 \mathrm{msec})$ in Experiment 3.

We ran a $2 \times 2$ ANOVA with block type (ignore vs. report) and SOA (200 vs. $800 \mathrm{msec})$ as within-subjects variables. The results are depicted in Figure 7.

The result pattern differed strongly from the ones found in Experiments 1 and 2. First, numerically, saccade latencies were more than one fourth lower than in the other two experiments (mean saccade latency was $162.2 \mathrm{msec}$ in Experiment 3, as compared with $226.05 \mathrm{msec}$ for the one-letter condition in Experiment 1 and 236.59 msec for the one-letter condition in Experiment 2). The impression that this decrease was indeed essential was supported by significant independent $t$ tests between the mean saccade latency in Experiment 3 and that in the one-letter condition in Experiment 1 or 2, respectively. Since Levene's test revealed unequal error variances for both tests $(p<$ .05 for Experiment 1 vs. Experiment 3, and $p<.001$ for Experiment 2 vs. Experiment 3), corrected degrees of freedom were used $[t(8.968)=5.744, p<.001$, for Experiment 1 vs. Experiment 3, and $t(24.318)=7.646, p<$ .001 , for Experiment 2 vs. Experiment 3]. Second, unlike in Experiments 1 and 2, only the main effects reached significance. Saccade latency was longer in the report condition $(165 \mathrm{msec})$ than in the ignore condition $(159 \mathrm{msec})$ $[F(1,11)=6.724, p<.05]$ and longer in the short-SOA

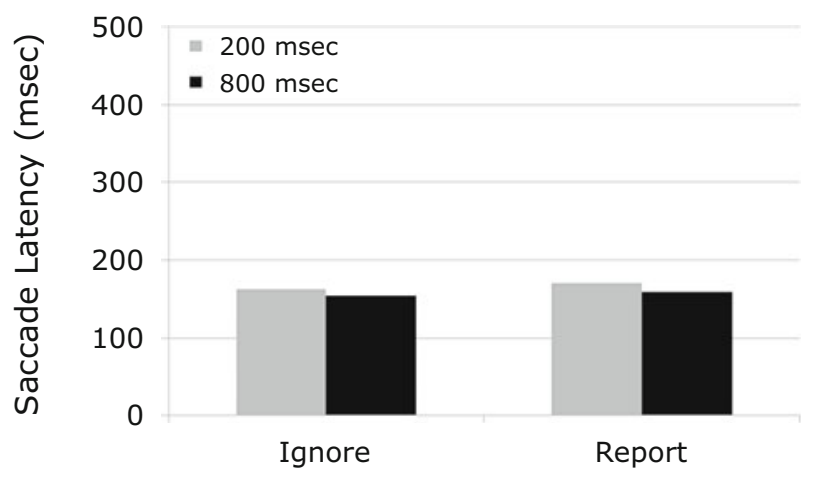

Figure 7. Mean saccade latencies for block type (ignore vs. report) and stimulus onset asynchrony ( $200 \mathrm{vs} .800 \mathrm{msec})$ in Experiment 3. 
condition $(167 \mathrm{msec})$ than in the long-SOA condition $(158 \mathrm{msec})[F(1,11)=12.927, p<.01]$. The interaction between block type and SOA did not reach significance $(F<1)$. Third, although significant, as compared with the other two experiments, the SOA difference and the reportignore difference were very small (a mean difference of 6 msec between the two SOAs and a mean difference of $9 \mathrm{msec}$ between the report and the ignore conditions).

The result pattern of Experiment 3 does not support an involvement of central attentional processes in the control of reflexive saccades. When stimuli from different modalities were used for both tasks, saccade latencies were lower than when both stimuli were visual; interference between the tasks was rather small, although significant; and more important, the interference (Diff SOA $_{\text {A }}$ ) did not increase in the report condition. The small interference effects might be explained by assuming that the auditory stimulus generated a brief orientation reflex that cannot be suppressed voluntarily and decays over time.

\section{GENERAL DISCUSSION}

The aim of the present experiments was to investigate whether central attentional processing is involved in the control of stimulus-driven saccades. We adapted a dualtask procedure used by Jolicœur and Dell'Acqua (1998), such that in the first two experiments, T1 required either identifying or ignoring briefly presented and masked letter stimuli, whereas T2, following T1 after variable SOAs, required a speeded saccade to a peripheral visual target. Following the logic of Jolicœur and Dell'Acqua, we manipulated - in addition to block type (report/ignore) and SOA - the amount of information for STC by varying the number of letters. On the basis of CIT, we expected a large RT difference between SOAs in the report condition, and this difference was supposed to be amplified for the three-letter condition, as compared with the one-letter condition. The result pattern found in Experiment 1 with constant letter presentation durations across the letter conditions matched these considerations, showing an increase of saccade latency difference in the report condition between short and long SOAs ( Diff $_{\mathrm{SOA}}$ ) for the three-letter condition, as compared with the one-letter condition. The same was true for Experiment 2 with varying presentation durations but comparable performance levels across letter conditions. However, in both experiments, we also found a significant Diff SOA $_{\text {in }}$ in the ignore condition. In Experiment 3, we substituted tones for T1-letters in order to test for the possibility that visual, modality-specific interference effects may have caused the result pattern found in Experiments 1 and 2. This seems indeed to be the case. Although we found a small difference between SOAs, this difference did not increase from the ignore to the report condition.

This data pattern seems to be evidence against an involvement of central attentional processes in the control of reflexive saccades, since Jolicœur and Dell'Acqua (1998) have found interference using different modalities and manual responses (buttonpresses) as the dependent variable. Why, then, did we not find central interference effects for saccades? The reason might be that our second stimulus (the ST) was able to specify the adequate response directly. The location of the stimulus was sufficient to program the saccade endpoint by a preceding spatial attention shift (e.g., Deubel \& Schneider, 1996), whereas in Jolicœur and Dell'Acqua's (1998) experiments, the identity of the auditory stimulus had to be recoded according to its pitch in order to specify the adequate manual response. This interpretation fits well with findings of Pashler et al. (1993), who combined a speeded tone discrimination task with a speeded saccade task. They found only a slight increase in saccade latencies with short SOAs when the task was to make a saccade toward a single visual stimulus or to a visual stimulus defined by its color (the relevant color remained constant across the trials). In both cases, a direct shift of spatial attention to the future ST location was able to specify the saccade. However, the increase was stronger when a central color patch indicated which of two stimulus locations constituted the ST. In this case, the arbitrary mapping between the stimulus color (red or green) and the saccade direction (left or right) prevented a direct saccade parameter specification (see Neumann, 1990) by a preceding spatial attention shift.

If the result pattern in Experiments 1 and 2 with two visual stimuli is not due to the involvement of central attention, how can it be explained? An alternative explanation needs to cover the finding of a longer saccade latency with short than with long SOAs in the report condition and the even stronger difference for the three- than for the oneletter condition. In addition, the latency difference between SOAs in the ignore condition needs to be explained as well. Given the lack of substantial Task 1 dependent interference in Experiment 3, in our view the biased competition (BC) framework of attention (Desimone \& Duncan, 1995) seems to be suitable. Proponents of BC assume that "objects" compete for selection at the neural level and that selection can be biased by bottom-up factors (saliency), as well as by top-down factors (such as task demands). As a result of $\mathrm{BC}$, one or several winners are selected for perception (e.g., verbal report) and action (e.g., speeded motor response). We adopt a specific approach to BC for selection in vision - namely, the theory of visual attention (TVA; Bundesen, 1990; Bundesen, Habekost, \& Kyllingsbæk, 2005). According to the TVA, the competition between visual objects is determined by their attentional weights. The higher an object's weight, the better its chance to win the competition. Attentional weights normalize to 1 ; that is, the more objects compete, the lower the relative attentional weight of the individual object, and the less probable its selection is. Because TVA in its current version (Bundesen \& Habekost, 2008) refers neither to saccade control nor to persistence of attentional weights after stimulus disappearance, we add two assumptions to the theoretical framework. First, attentional weights regulate the competition between potential STs (Wischnewski, Belardinelli, Schneider, \& Steil, in press). Therefore, the relative attentional weight of an ST determines its processing priority and should thus be inversely related to saccade latency (the higher the relative attentional weight, the shorter the saccade latency). Second, after the disappearance of an object, the magnitude 
of its attentional weight slowly decays to zero within the iconic time range.

Given these two assumptions of an extended TVA, the result pattern in our experiments can be explained as follows. First, the longer saccade latencies for short than for long SOAs in the report condition are due to the relative attentional weight of the ST being decreased by the competing weight of T1. This decreased ST weight leads to prolonged saccade latencies. In case of the long SOA, the weight of T1 already has dissolved. Therefore, T1 cannot compete with the ST; the relative weight of the ST is high, and saccade latency is short. Second, the new key finding of our study - namely, that the saccade latency difference between SOAs in the report condition was more pronounced for three letters than for one letter-can be explained, since each letter is relevant and receives an absolute attentional weight of the same value. Following the weight equation of the TVA (Bundesen, 1990), the relative attentional weight of the ST is decreased more strongly by three absolute weights of three letters than by one absolute weight of one letter, and thus, saccade latency increases more strongly for three letters. Third, in contrast to CIT, an extended TVA can explain the higher saccade latencies for short than for long SOAs in the ignore condition. Following the standard TVA (Bundesen \& Habekost, 2008), even irrelevant objects with an abrupt onset receive an attentional weight larger than zero. Thus, the absolute weights of irrelevant letters still reduce the relative attentional weight of the ST at short SOAs and lead to an increase of saccade latencies. However, at long SOAs, the weight of irrelevant letters already has decayed to zero. However, one puzzling finding escapes the extended TVA explanation. In the ignore condition, the saccade latency increase from long to short SOAs is the same for one and for three letters. The following assumption may serve as an explanation. By combining the Gestalt laws of common fate and proximity with the standard TVA (see also Bundesen \& Habekost, 2008), three letters that are irrelevant and appear with a common onset and in immediate proximity might be treated as a single perceptual unit during the first bottom-up wave of processing. If so, a corresponding single attentional weight should be computed in the one- as well as in the three-letter condition. Consequently, the relative attentional weight of the ST in the ignore condition should be the same for both letter conditions, and the saccade latency increase for short SOAs should be the same for one and for three letters. Surely, further research is needed to determine whether these assumptions extending the TVA to saccade control are justified.

\section{AUTHOR NOTE}

This work was supported by a grant of the Cluster of Excellence "Cognitive Interaction Technology" (CITEC) to W.X.S. and E.C. We are very grateful to Kathi Seidl and Jan Zwickel for essential contributions in developing the experimental paradigm introduced in this article. We thank Yaffa Yeshurun, Roberto Dell'Acqua, Claus Bundesen, and an anonymous reviewer for their helpful comments on an earlier draft. Correspondence concerning this article should be addressed to E. Carbone, Abteilung für Psychologie, Universität Bielefeld, Postfach 100131, D-33501 Bielefeld, Germany (e-mail: elena.carbone@uni-bielefeld.de).

\section{REFERENCES}

Awh, E., Armstrong, K. M., \& Moore, T. (2006). Visual and oculomotor selection: Links, causes and implications for spatial attention. Trends in Cognitive Sciences, 10, 124-130.

Brisson, B., \& Jolicceur, P. (2007). Electrophysiological evidence of central interference in the control of visuospatial attention. Psychonomic Bulletin \& Review, 14, 126-132.

Bundesen, C. (1990). A theory of visual attention. Psychological Review, 97, 523-547.

Bundesen, C., \& Habekost, T. (2008). Principles of visual attention. Oxford: Oxford University Press.

Bundesen, C., Habekost, T., \& Kyllingsbæe, S. (2005). A neural theory of visual attention: Bridging cognition and neurophysiology. Psychological Review, 112, 291-328.

Chun, M. M., \& Potter, M. C. (1995). A two-stage model of multiple target detection in rapid serial visual presentation. Journal of Experimental Psychology: Human Perception \& Performance, 21, 109-127.

Dell'Acqua, R., Sessa, P., Joliceeur, P., \& Robitaille, N. (2006) Spatial attention freezes during the attentional blink. Psychophysiology, 43, 394-400.

Desimone, R., \& Duncan, J. (1995). Neural mechanisms of selective visual attention. Annual Review of Neuroscience, 18, 193-222.

Deubel, H., \& Schneider, W. X. (1996). Saccade target selection and object recognition: Evidence for a common attentional mechanism. Vision Research, 36, 1827-1837.

FindLAY, J. M. (2009). Saccadic eye movement programming: Sensory and attentional factors. Psychological Research, 73, 127-135.

JOLICEUR, P. (1998). Modulation of the attentional blink by on-line response selection: Evidence from speeded and unspeeded Task $\mathrm{deci-}$ sions. Memory \& Cognition, 26, 1014-1032.

JoLICGUR, P. (1999a). Concurrent response-selection demands modulate the attentional blink. Journal of Experimental Psychology: Human Perception \& Performance, 25, 1097-1113.

JoLICEUR, P. (1999b). Dual-task interference and visual encoding. Journal of Experimental Psychology: Human Perception \& Performance, 25, 596-616

Joliceur, P., \& Dell'Acqua, R. (1998). The demonstration of shortterm consolidation. Cognitive Psychology, 36, 138-202.

Kahneman, D. (1973). Attention and effort. Englewood Cliffs, NJ: Prentice Hall.

Kustov, A. A., \& Robinson, D. L. (1996). Shared neural control of attentional shifts and eye movements. Nature, 384, 74-77.

Neumann, O. (1990). Direct parameter specification and the concept of perception. Psychological Research, 52, 207-215.

Pashler, H. (1994). Dual-task interference in simple tasks: Data and theory. Psychological Bulletin, 16, 220-244.

Pashler, H., Carrier, M., \& Hoffman, J. (1993). Saccadic eye movements and dual-task interference. Quarterly Journal of Experimental Psychology, 46A, 51-82.

SCHNEIDER, W. X. (1995). VAM: A neuro-cognitive model for visual attention control of segmentation, object recognition, and space-based motor action. Visual Cognition, 2, 331-375.

Schneider, W. X., \& Deubel, H. (1995). Visual attention and saccadic eye movements: Evidence for obligatory and selective spatial coupling. In J. M. Findlay, R. Walker, \& R. W. Kentridge (Eds.), Eye movement research: Mechanisms, processes, and applications (pp. 317-324). Amsterdam: Elsevier.

Schneider, W. X., \& Deubel, H. (2002). Selection-for-perception and selection-for-spatial-motor-action are coupled by visual attention: A review of recent findings and new evidence from stimulus-driven saccade control. In W. Prinz \& B. Hommel (Eds.), Common mechanisms in perception and action: Attention and performance XIX (pp. 609627). Oxford: Oxford University Press.

Wischnewski, M., Belardinelli, A., Schneider, W. X., \& Steil, J. J. (in press). Where to look next? Combining static and dynamic proto-objects in a TVA-based model of visual attention. Cognitive Computation.

(Manuscript received December 18, 2009; revision accepted for publication June 6, 2010.) 